\title{
采用人类视觉对比机制的红外弱小目标检测
}

\author{
刘旭 ${ }^{1,2,3}$, 崔文楠 ${ }^{1,2}$ \\ (1. 中国科学院 智能红外感知重点实验室, 上海 200083；2. 中国科学院 上海技术物理研究所, 上海 200083; \\ 3. 中国科学院大学, 北京 100049)
}

\begin{abstract}
摘要: 针对复杂背景下红外弱小目标检测难题, 提出一种基于人类视觉系统对比机制的红外弱小目标 检测算法。首先, 对红外图像进行预处理, 通过中值滤波去除红外图像中的孤立噪声点。然后对处理 后的图像进行高斯函数差分滤波处理，抑制图像中大面积高亮区域。最后，通过改进的基于局部对比 度方法去除高亮边缘区域, 消除高疑似目标, 最终实现对复杂背景下红外弱小目标的检测。实验表明: 相较于传统的 LCM 算法、Top-hat 算法、TDLMS 算法和 Infrared Patch-Image Model 算法等, 该算法 在虚警率、正确检测率、检测时间等方面更有优势，具有检测率高、虚警率低、鲁棒性好、运行时间 短的特点。
\end{abstract}

关键词：弱小目标检测；高斯函数差分滤波器；局部对比度方法；红外图像

中图分类号：TP751.1 文献标识码：A 文章编号：1001-8891(2020)06-0559-07

\section{Infrared-Image-Based Detection of Dim and Small Targets Using Human Visual Contrast Mechanism}

\author{
LIU Xu ${ }^{1,2,3}$, CUI Wennan ${ }^{1,2}$ \\ (1. Key Laboratory of Intelligent Infrared Perception, Chinese Academy of Sciences, Shanghai 200083, China; \\ 2. Shanghai Institute of Technical Physics, Chinese Academy of Sciences, Shanghai 200083, China; \\ 3. University of Chinese Academy of Sciences, Beijing 100049, China)
}

\begin{abstract}
In this paper, an infrared-image-based algorithm is proposed for the detection of dim and small targets in complex backgrounds. The proposed algorithm is based on the contrast mechanism of the human visual system. First, an infrared image was preprocessed, and isolated noise points in the image were removed via median filtering. The processed image was then subjected to difference-of-Gaussians filtering to suppress large-area highlighted areas in the image. Finally, an improved local contrast algorithm was used to remove the highlighted edge regions and eliminate the high suspect target to achieve the detection of dim and small targets in complex backgrounds using infrared images. Experimental results show that compared with the traditional LCM algorithm, top-hat algorithm, TDLMS algorithm, and infrared patch-image model, the proposed algorithm is more advantageous with regard to the false alarm rate, correct detection rate, detection time, etc. It also has the characteristics of a high detection rate, low false alarm rate, good robustness, and short running time.
\end{abstract}

Key words: dim and small target detection, difference of Gaussians, local contrast method, infrared image

\section{0 引言}

近年来随着计算机视觉和红外成像技术的发 展, 红外成像被广泛应用于人脸识别、精确制导和 医学成像等各个领域 ${ }^{[1]}$, 而弱小目标的检测与跟踪一 直是红外成像探测系统的核心技术之一，目标检测
算法会直接影响到整个探测系统的性能 ${ }^{[2]}$ 。在背景复 杂的实际场景中, 由于成像距离远、长距离大气程 辐射干扰造成的影响，导致目标成像面积小、信号 弱, 经常具有形状信息缺失、纹理特征不明显、低 信噪比等特点, 常被淹没在亮度较高且高疑似目标 较多的图像背景中。 
针对复杂背景下的弱小目标检测问题, 大量基于 不同原理的检测算法被提出, 这些算法可归为两类: 检测前跟踪 (track before detect, TBD) 和跟踪前检测 (detect before track, DBT) 算法。检测前跟踪即对多 帧图像中较多疑似轨迹进行跟踪, 跟踪前检测即根据 门限阈值对每帧图像检测。其中跟踪前检测算法大致 分为基于滤波、基于人类视觉系统 (human visual system, HSV) 和基于时域检测 3 类。其中人类视觉系 统的工作机制包括：(1) 对比机制 (contrast mechanism): 模拟人眼以局部对比度信息为依据处理 视场信息; (2)自适应尺度匹配机制 (size-adaptive process ): 模拟人眼搜索目标时快速捕捉与目标尺度 相似的 “小面积” 区域, 而非像通常算法逐像素处理 图像; (3)视场弹出机制 (pop-out phenomenon): 模拟 人眼优先关注最突出（局部对比度最大）区域, 直接 舍弃不突出区域; (4) 回归抑制机制 (inhibition-of-return): 模拟人眼对之前关注过的突出 区域不再重复关注, 直接搜索下一个次突出区域直至 该区域低于某阈值搜索结束。

目前, 大量基于局部对比度机制的目标检测算法 被提出。Kim 等 ${ }^{[3]}$ 在 2009 年使用 LoG (Laplacian of Gaussian）滤波器对图像进行滤波实现小目标检测, 即先对图像进行高斯滤波, 再用 Laplace 算子进行边 缘检测, 由此形成用于极值点检测的 LoG 算子。Wang 等 ${ }^{[4]}$ 在 2012 年使用更为简便的高斯函数差分 (difference of Gaussian, DoG) 滤波器, DoG 滤波器是 由两个不同参数的二维高斯函数进行求差, 得到中心 为正、四周为负的无方向性的卷积滤波器, 可模拟人 类视觉神经细胞的感受野模式。Chen 等 ${ }^{[5]}$ 在 2013 年 提出基于局部对比度的方法 (local contrast method, LCM）的目标检测算法, 算法采用中心灰度最大值和 周围灰度平均值作为增强系数来对目标增强。同时, 算法将周围邻域按方向划为 8 个子块, 具有方向敏感 性。Xie 等 ${ }^{[6]}$ 在 2014 年提出 ACSDM (accurate center-surround difference measure) 算法, 在求对比度 时采用差值形式, 有效消除大面积高亮背景区域, 并 同时考虑对比度的方向性问题, 消除普通背景边缘干 扰。传统的基于局部对比度机制的目标检测算法在信 噪比较高、背景单一时有较为理想的检测结果, 但其 缺点在于部分检测算法未考虑方向性, 无法有效区分 真实目标和普通背景边缘, 而考虑方向性的算法需要 逐像素计算, 实时性较差。

本文针对复杂背景下的红外弱小目标检测问题， 提出一种基于人类视觉对比机制的弱小目标检测算 法。首先, 对红外图像进行中值滤波预处理去除孤立
噪声点, 然后进行 DoG 滤波处理, 接着使用改进的 基于局部对比度方法处理, 最终阈值分割得到检测结 果。整个算法流程如图 1 所示。

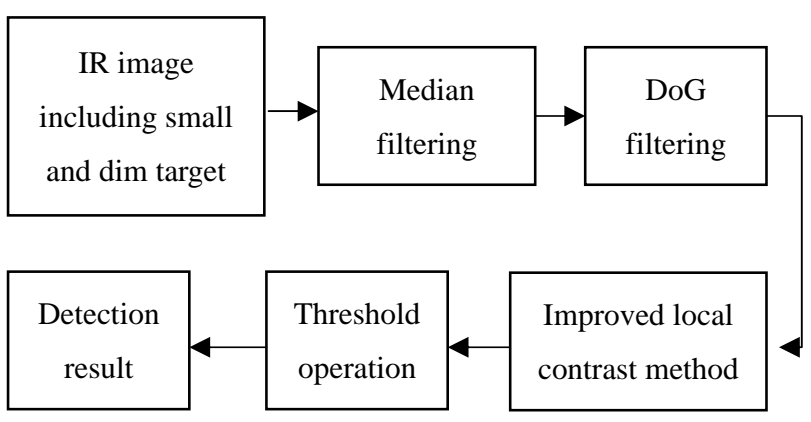

图 1 弱小目标检测流程图

Fig.1 Dim and small target detection flow chart

\section{1 红外弱小目标特性分析}

目前红外成像系统在空间分辨率方面已经做到 或接近理论上的极限值, 但由于实际应用场景中距离 过远, 红外目标的成像尺寸在图像中所占比例较小。 根据国际光学工程学会 (society of photo-optical instrumentation engineers, SPIE) 对于红外弱小目标的 定义, 对于 $256 \times 256$ 的图像, 成像尺寸小于总像素 数的 $0.12 \%$ 的目标为弱小目标 ${ }^{[7]}$ 。

包含弱小目标的红外图像可认为由目标、噪声和 背景组成, 模型如下:

$$
f(x, y)=f_{\mathrm{T}}(x, y)+f_{\mathrm{B}}(x, y)+n(x, y)
$$

式中: $f(x, y)$ 为红外图像; $f_{\mathrm{T}}(x, y)$ 为弱小目标; $f_{\mathrm{B}}(x, y)$ 为背景杂波; $n(x, y)$ 为噪声。其中, 弱小目标 $f_{\mathrm{T}}(x, y)$ 和背景区域相关性较差, 表现为一个个孤立的亮斑, 属于高频成分; 背景杂波 $f_{\mathrm{B}}(x, y)$ 除了边缘区域有少量 高频成分, 一般是连续的大块区域, 大部分属于低频 成分; 噪声 $n(x, y)$ 的空间分布比较随机, 帧间相关性 较小。

\section{2 高斯函数差分滤波器}

复杂背景下的远距离弱小目标非常接近点目标, 经过光学点扩散函数成像后其在焦平面上呈高斯斑 点, 成像函数表达式如下 ${ }^{[8]}$ :

$$
g_{\mathrm{T}}(r)=A \exp \left(-\frac{x^{2}+y^{2}}{2 \sigma_{0}^{2}}\right)
$$

式中: $\sigma_{0}$ 为目标尺度; $A$ 为目标幅值; $r$ 为位置矢量, $r=(x, y), x 、 y$ 为二维平面横纵坐标。

DoG 滤波器是一种基于人类视觉对比度机制的 带通滤波器, 其表达式如下: 


$$
f\left(u, v, \sigma_{1}, \sigma_{2}\right)=\frac{1}{2 \pi \sigma_{1}^{2}} \mathrm{e}^{-\frac{u^{2}+v^{2}}{2 \sigma_{1}^{2}}}-\frac{1}{2 \pi \sigma_{2}^{2}} \mathrm{e}^{-\frac{u^{2}+v^{2}}{2 \sigma_{2}^{2}}}
$$

式中: $u 、 v$ 分别为二维平面横、纵坐标; $\sigma_{1}$ 和 $\sigma_{2}$ 分 别为两个二维高斯函数的尺度参数, 并且 $\sigma_{1}<\sigma_{2}$, 其 几何空间分布示意图如图 2 所示, 其中 $\sigma_{1}=2, \sigma_{2}=$ 10 即本文算法设定参数。

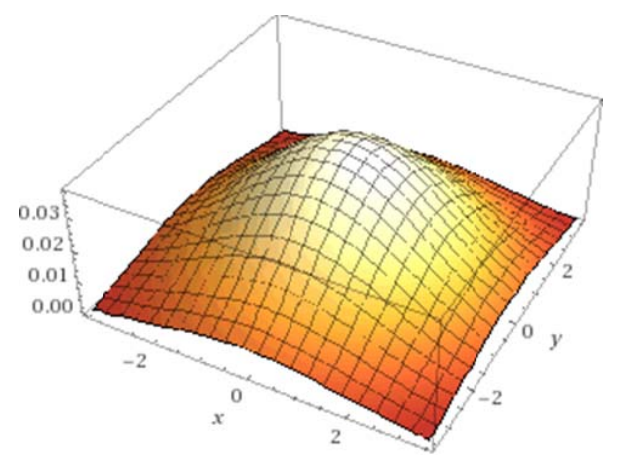

图 2 DoG 函数的几何空间分布

Fig.2 Geometric spatial distribution of DoG function

显然, 当 DoG 算子中心区域对四周均为背景的 弱小目标区域进行卷积滤波时, 响应值较大; 当 DoG 算子对背景区域进行卷积滤波时, 无论背景区域亮度 如何, 响应值均为 0 。当 DoG 算子尺度与高斯斑点尺 度相等时, 响应值最大。因此, DoG 算子可以有效地 消除红外图像中低频大面积高亮背景和高频噪声干 扰，保留弱小目标。

然而, 经典的 DoG 算子是圆形对称的不具有方 向敏感性, 其对背景边缘较为敏感, 即所谓的边缘效 应 $^{[9]}$ 。因此, 仅靠 DoG 滤波器不足以将复杂背景下的 弱小目标提取出来, 需要对红外图像作进一步处理。

\section{3 改进的 LCM 算法}

LCM 算法的核心思想是利用红外弱小目标亮度 高于周围背景且为孤立点的特性, 将中心最大灰度值 与周围背景平均灰度值比值作为目标灰度值的增强 系数, 最终达到增强目标抑制背景的目的。其具体原 理如下:

首先通过窗口滑动得到相应区域 $v$, 再将该区域 分割为 9 个子块如图 3 所示, 中心子块标号为 0 , 其 他 8 个子块依次标号为 $1,2,3, \cdots, 7,8$, 平均灰 度值依次为 $m_{0}, m_{1}, m_{2}, \cdots, m_{7}, m_{8}$, 标号为 0 的 子块记为 $u, u$ 内所有像素点灰度值的最大值为 $L_{\mathrm{n}}$ 。

然后按照表达式(4)计算 $c_{\mathrm{n}}$ 并替代中心子块 $u$ 的 灰度值:

$$
C_{\mathrm{n}}=\min \frac{L_{\mathrm{n}}{ }^{2}}{m_{i}}
$$

最后按照从左到右、从上到下的顺序逐个像素遍 历整幅图像，阈值分割后即可得到结果。

LCM 算法具有方向性, 但其仍有一定缺陷。首 先, 逐个像素计算并比较 8 个方向的对比度信息导致 算法耗时较长; 其次, 如图 4 所示, 算法检测到的目 标为右侧框选区域中的建筑边缘, 而真实目标为左侧 框选区域中的无人机, 结果显示对相较于目标更亮且 所占像素较少的高亮边缘, 在高疑似目标较多的复杂 背景下 LCM 算法实际处理效果并不理想。

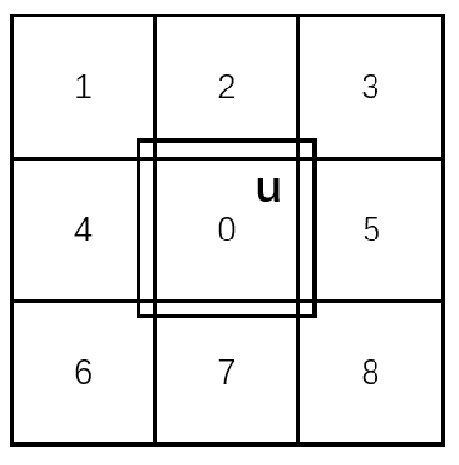

图 3 滑动窗口 $\mathrm{v}$ 及其子块

Fig.3 Sliding window $\mathrm{v}$ and its sub-blocks

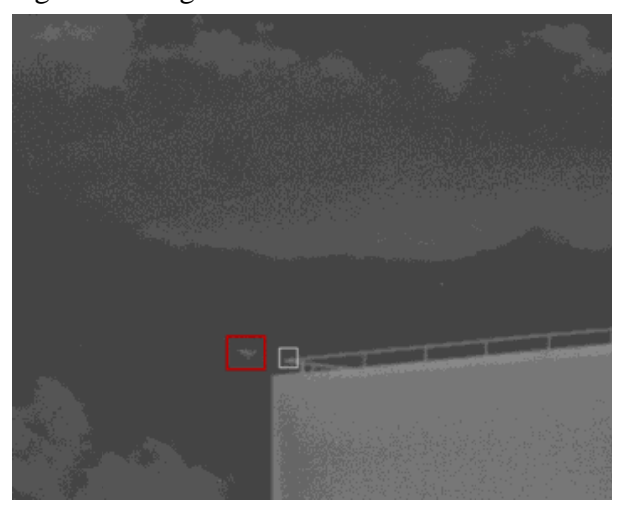

图 4 LCM 算法处理结果

Fig.4 LCM algorithm processing results

考虑到上述 LCM 算法不足之处，Chen 等 ${ }^{[5]}$ 提出 多尺度计算方式提高算法准确率, 但不断改变窗口大 小会导致算法耗时较长; Han 等 ${ }^{[10]}$ 通过对图像滤波预 处理并划分子块的计算方式降低虚警率并减少算法 耗时; Qin 等 ${ }^{[11]}$ 提出了一种新型的局部对比度测量算 法 (novel local contrast method, NLCM), 引入自定义 的均值和方差; Shi 等 ${ }^{[12]}$ 先通过一种改进的高增压滤 波器增强高频信号并抑制低频信号, 然后再使用 MLCM (multiscale local contrast measure) 算法处理 图像。

本文提出了一种改进的基于局部对比度算法。首 先, 由于 LCM 算法逐个像素点移动窗口的计算方式 耗时较长, 因此理论上移动步长大于 1 个像素点即可 
节省算法时间; 其次, 利用弱小目标亮度通常高于周 围环境, 尤其经过 DoG 算子滤波后此特征更加明显 的特性, 仅对亮度高于经验值的像素点进行 LCM 相 关计算, 进一步节省算法耗时; 最后, 利用高疑似目 标 (建筑物边缘、云层边缘等) 周围环境通常较为复 杂、高频成分较多的特点, 采用多尺寸联合计算的方 式, 对像素点周围高频成分像素点占比进行计算, 高 于经验值的像素点将被舍弃, 不再进行 LCM 相关计 算, 有效降低虚警率, 剔除疑似目标。算法具体流程 如下:

步骤 1: 待处理图像记为 $I$, 通过窗口滑动得到 大小为 $3 K \times 3 K$ 的相应区域 $v$, 再将该区域分割为 9 个 $K \times K$ 大小的子块, $u$ 内所有像素点灰度值的最大 值为 $L_{\mathrm{n}}$, 当 $L_{\mathrm{n}}$ 大于阈值 $\mathrm{Th}_{1}$ 时继续步骤 2 , 其中 $\mathrm{Th}_{1}$ 表达式如下:

$$
\mathrm{Th}_{1}=\bar{I}+k_{1} \times \sigma_{I}
$$

式中: $\bar{I}$ 为图像 $I$ 像素平均值; $\sigma_{I}$ 为图像 $I$ 像素标准 差, $k_{1}$ 根据经验通常取 $3 \sim 5$ 。

步骤 2: 分割得到以 $u$ 为中心的 $5 K \times 5 K$ 大小的 子块 $w$ 如图 5 所示, 计算中心子块 $u$ 的环境复杂程度 cl, 其表达式如下:

$$
\mathrm{cl}=\frac{\sum_{(i, j) \in c_{v}^{*}} f(i, j)}{N(i, j)}
$$

式中: $f(i, j)$ 为子块 $w$ 二值化处理后 $(i, j)$ 处的灰度值; $N(i, j)$ 为子块 $w$ 内的像素点个数。

步骤 3: 当 $\mathrm{cl}$ 小于阈值 $\mathrm{Th}_{2}$ 时继续步骤 4 , 否则 子块 $u$ 内所有像素的灰度值置为 0 。其中 $\mathrm{Th}_{2}$ 表达式 如下:

$$
\mathrm{Th}_{2}=k_{2} \times \overline{I^{\mathrm{bw}}}
$$

式中: $\overline{I^{\mathrm{bw}}}$ 为图像 $I$ 二值化处理后像素的平均值; $k_{2}$ 根据经验通常取 $0.5 \sim 0.7$ 。

步骤 4: 按照表达式(4)计算 $c_{\mathrm{n}}$ 并替代中心子块 $u$ 的灰度值。

步骤 5: 以一定步长在红外图像内选择子块窗口 $v$, 直至整幅图像遍历结束。

依据目标大小设定合适的窗口尺寸 $K$ 和移动步 长是算法能否得到理想结果的关键, 通常窗口尺寸 $K$ 略大于目标尺寸, 依据数据集图像尺寸本文 $K$ 设为 10。在窗口尺寸选定前提下, 目标与滑动窗口的关系 如图 6 所示, 显然, 滑动窗口若想在移动步长较大的 前提下尽可能覆盖目标, 步长宜设为 $K$ 左右。

除此之外, 阈值 $k_{1} 、 k_{2}$ 的取值对算法性能亦有影 响。 $k_{1}$ 即 $\mathrm{Th}_{1}$ 过小可能导致参与 LCM 算法计算的像
素点过多，增加算法耗时; $k_{1}$ 过大则可能导致真实目 标像素点无法参与 LCM 算法计算, 影响算法准确率。 $k_{2}$ 即 $\mathrm{Th}_{2}$ 过小可能导致真实目标因邻域存在杂波被误 判为虚警目标而被剔除; $k_{2}$ 过大则可能导致高疑似目 标无法被有效剔除。算法处理过程及最终结果如图 7 所示。

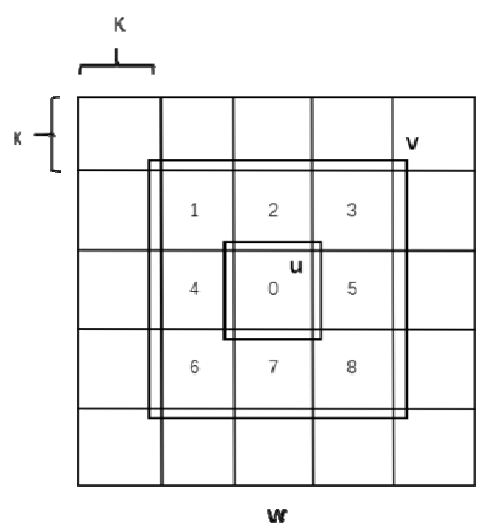

图 5 滑动窗口 $w$ 及其子块

Fig.5 Sliding window $w$ and its sub-blocks

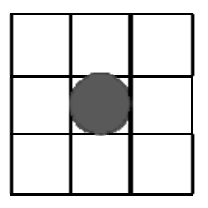

(1)

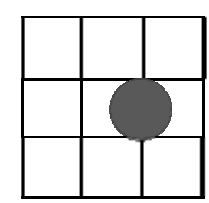

(2)

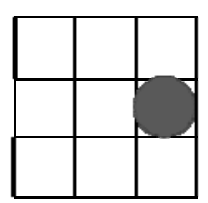

(3)
图 6 滑动窗口与弱小目标尺寸比较

Fig.6 Comparison of the size of sliding window and small and dim target

\section{4 实验结果}

为验证本文算法性能, 本文在配置为 $2.2 \mathrm{GHz}$ Intel ${ }^{\circledR}$ Core $^{\mathrm{TM}} \mathrm{i7}-8750 \mathrm{H}$ 处理器、4 G 独显 GTX1050Ti、 $8 \mathrm{G}$ 内存的 OMEN by HP Laptop 15-dc0006TX 电脑上 对若干实际环境中拍摄的红外图像进行仿真实验, 系 统环境为 64 位 Windows 10 家庭中文版, 所用软件为 MATLAB R2018a。弱小目标信号质量通常可以用信 噪比 SNR 和信杂比 SCR 描述, 同时为衡量算法有效 性, 设置了正确检测率 $P_{\mathrm{d}}$ 、虚警率 $F_{\mathrm{a}} \mathrm{PR}$ 和算法耗时 t3 个性能指标参数, 其定义如下:

信噪比 SNR:

$$
\mathrm{SNR}=\mu_{\mathrm{t}} / \sigma_{\mathrm{b}}
$$

式中: $\mu_{\mathrm{t}}$ 表示目标区域的平均灰度; $\sigma_{\mathrm{b}}$ 表示背景区域 的标准差。

信杂比 SCR:

$$
\mathrm{SCR}=\left|\mu_{\mathrm{t}}-\mu_{\mathrm{b}}\right| / \sigma_{\mathrm{b}}
$$

式中: $\mu_{\mathrm{b}}$ 表示背景区域的平均灰度。

正确检测概率 $P_{\mathrm{d}}$ : 


$$
P_{\mathrm{d}}=\frac{\text { 正确检测的目标数 }}{\text { 总的真实目标数 }}
$$

虚警率 $F_{\mathrm{a}} \mathrm{PR}$ :

$$
F_{\mathrm{a}} \mathrm{PR}=\frac{\text { 错误检测的像素数 }}{\text { 整幅图像像素总数 }}
$$

图 8 为 5 种算法对 3 幅复杂场景下红外图像进行 目标检测的结果, 其中(a1)中两目标的 SNR 为 4.8824、 3.4537, SCR 为 2.1004、0.7285, (b1)目标的 SNR 为 2.1722, SCR 为 $0.1822,(\mathrm{c} 1)$ 目标的 SNR 为 2.177, SCR 为 0.4468 , 不同检测算法性能如表 1 所示。从图 8 和表 1 中可以看出, 当目标信噪比和信杂比较低时,
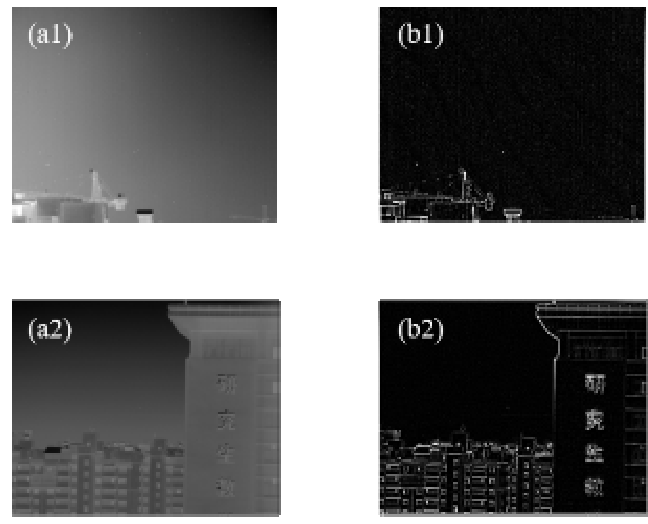

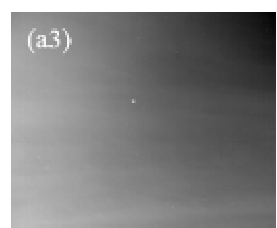

(a) Original image (a) 原始图像

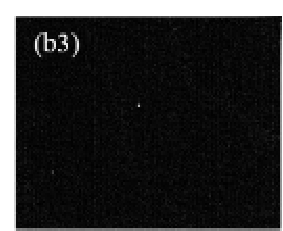

(b) DoG 滤波后结果
本文算法在得到较为理想的检测结果的同时, 虚警率 较低, 所用时间低于 $\mathrm{LCM}^{[5]}$ 和 $\mathrm{TDLMS}^{[13]}$ 算法, 与 Top-hat ${ }^{[14]}$ 和 Infrared Patch-Image Model ${ }^{[15]}$ 所用时间 相近。

表 2 为实验中不同算法对 103 幅实际拍摄的 640 $\times 512$ 图像的处理结果, 表 3 为实验中不同算法对 30 幅 $256 \times 200$ 图像 ${ }^{[16]}$ 的处理结果，结果表明本文算法 在所用时间较短的情况下, 可以有效剔除疑似目标, 保持较高的正确检测概率。
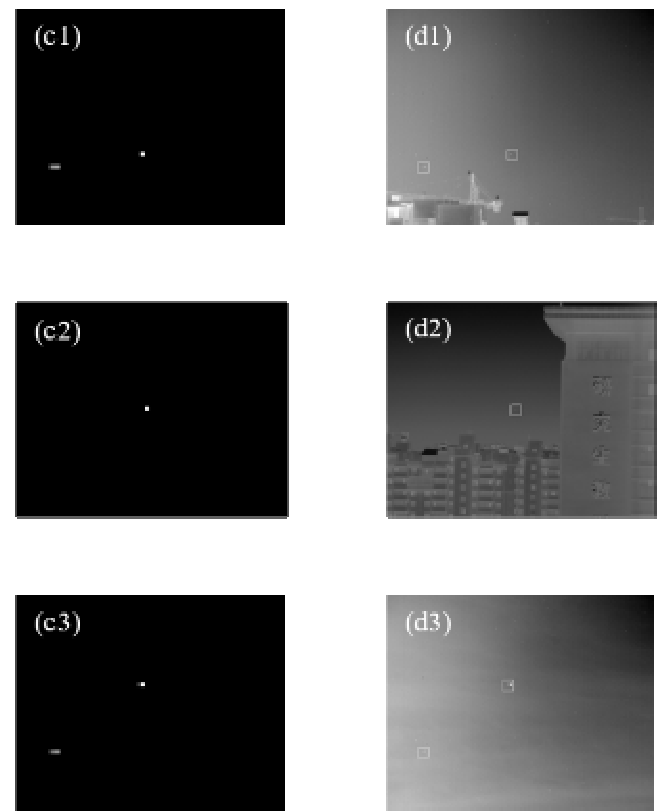

(c) LCM 算法处理后结果

(d) 检测结果

$\begin{array}{ll}\text { (c) LCM algorithm processed results } & \text { (d) Test results }\end{array}$
图 7 本文算法处理结果
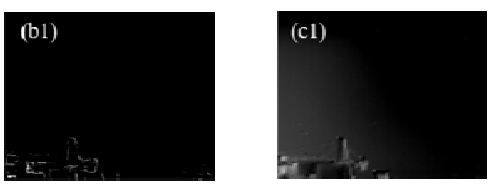

Fig.7 Proposed algorithm processing results
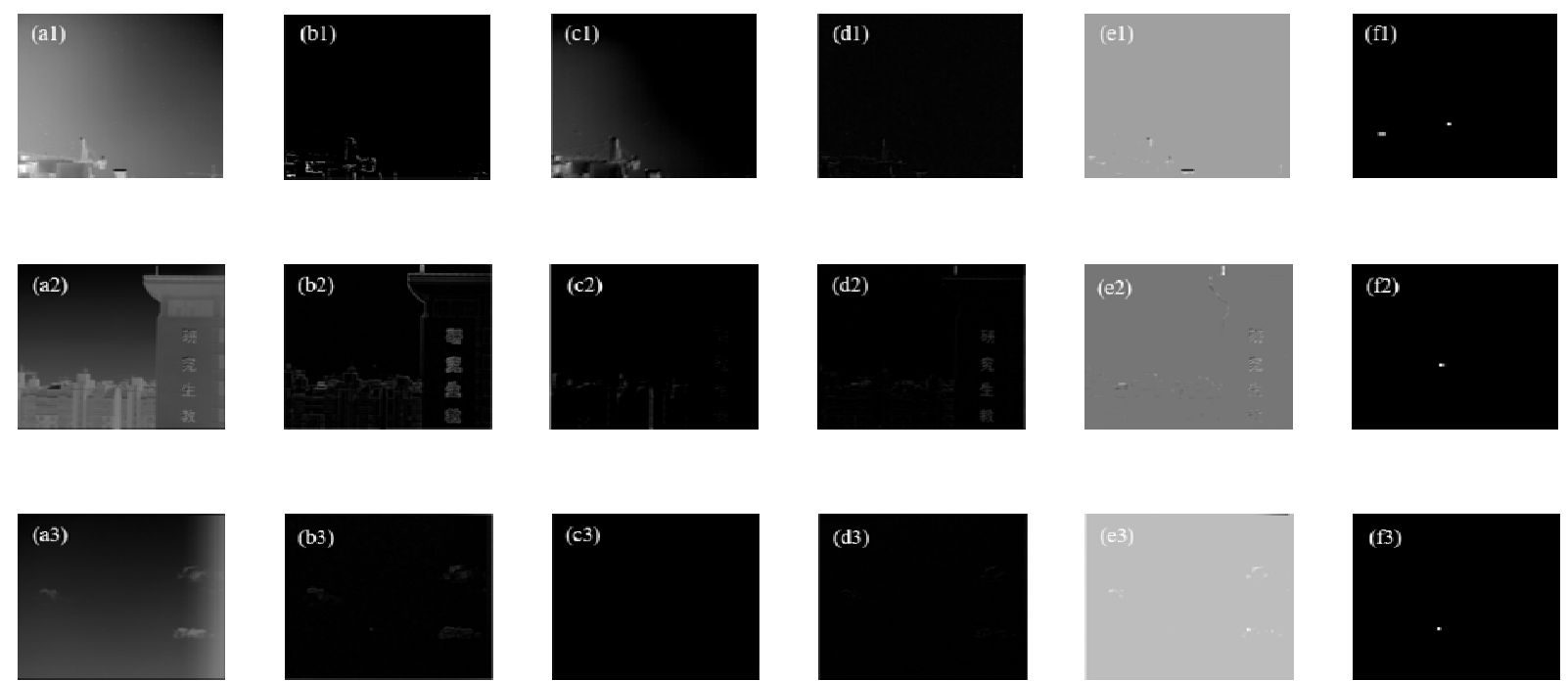

(a) 原始图像

(b) 局部对比度方法(c)

(a) Original image
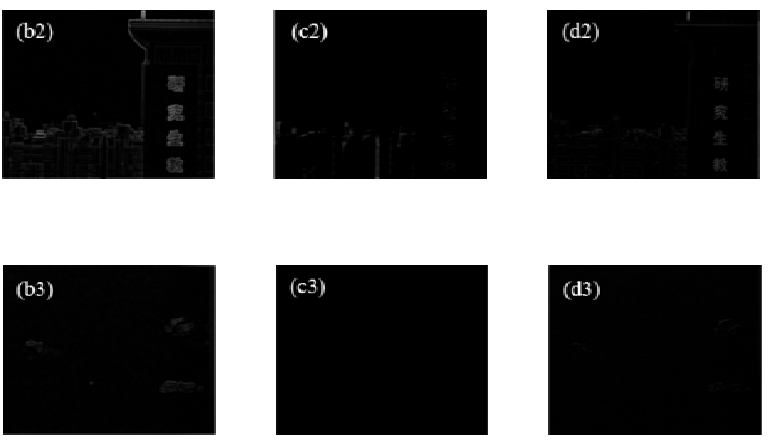
(b) LCM
(c) TDLMS
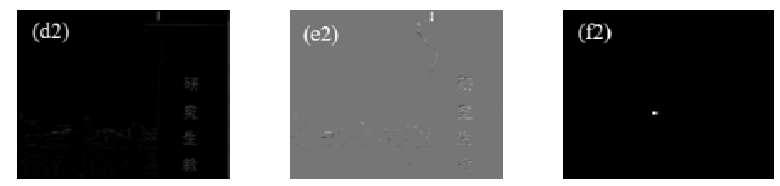

图 8 不同算法处理结果

Fig.8 Different algorithm processing results 
表 1 不同检测算法性能比较

Table 1 Performance comparison of different detection algorithms

\begin{tabular}{ccccccccccc}
\hline \multirow{2}{*}{ Algorithm } & \multicolumn{3}{c}{ Seq. 1} & \multicolumn{3}{c}{ Seq. 2} & \multicolumn{3}{c}{ Seq. 3} \\
\cline { 2 - 10 } & $P_{\mathrm{d}}$ & $F_{\mathrm{a} P R}$ & Timet/s & $P_{\mathrm{d}}$ & $F_{\mathrm{a}} \mathrm{PR}$ & Time t/s & $P_{\mathrm{d}}$ & $F_{\mathrm{a}} \mathrm{PR}$ & time t/s \\
\hline LCM & 0.5 & 0.0041 & 0.4223 & 1 & 0.0328 & 0.2248 & 0 & 0.0128 & 0.2186 \\
TDLMS & 1 & 0.0159 & 0.6960 & 0 & 0.0019 & 0.6321 & 0 & $9.460 \mathrm{e}-05$ & 0.6241 \\
$\begin{array}{c}\text { Top-hat } \\
\text { Infrared patch- }\end{array}$ & 1 & 0.0119 & 0.2340 & 0 & 0.0190 & 0.0774 & 0 & 0.0033 & 0.0317 \\
image model & 1 & 0.0019 & 9.4692 & 0 & 0.0054 & 11.6477 & 1 & 0.0028 & 10.8831 \\
This article & 1 & 0 & 0.1078 & 1 & 0 & 0.0635 & 1 & 0 & 0.0276 \\
\hline
\end{tabular}

\section{表 2 不同算法对多幅 $640 \times 512$ 图像的检测结果}

Table 2 Detection results of multiple images by different algorithms

\begin{tabular}{cccccc}
\hline Algorithm & LCM & TDLMS & Top-hat & Infrared patch-image model & This paper \\
\hline$P_{\mathrm{d}} / \%$ & 60.19 & 34.47 & 55.66 & 58.74 & 78.64 \\
$F_{\mathrm{a}} \mathrm{PR} / \%$ & 0.86 & 3.98 & 6.92 & 0.17 & 0.03 \\
Time/s & 0.254 & 0.579 & 0.016 & 9.449 & 0.051 \\
\hline
\end{tabular}

表 3 不同算法对多幅 $256 \times 200$ 图像的检测结果

Table 3 Detection results of multiple images by different algorithms

\begin{tabular}{cccccc}
\hline Algorithm & LCM & TDLMS & Top-hat & Infrared patch-image model & This paper \\
\hline$P_{\mathrm{d}} / \%$ & 56.67 & 53.33 & 33.33 & 100.00 & 90.00 \\
$F_{\mathrm{a}} \mathrm{PR} / \%$ & 20.68 & 10.03 & 30.18 & 0.0156 & 0.1893 \\
Time/s & 0.0561 & 0.1087 & 0.0008 & 2.5799 & 0.0142 \\
\hline
\end{tabular}

\section{5 结论}

针对复杂背景下红外弱小目标检测问题, 本文提 出一种基于人类视觉系统对比机制的弱小目标检测 算法。该算法通过 DoG 滤波器抑制了图像中的大面 积高亮区域, 再通过改进的 LCM 算法剔除高亮边缘 和高疑似目标, 解决了复杂背景下检测弱小目标时虚 警率较高的问题。上述实验表明：本文算法在低信噪 比、低信杂比的情况下, 有效降低了虚警率并保证算 法速度, 保证了较高的正确检测概率, 得到了较为理 想的检测结果, 解决了实际应用场景中环境复杂、目 标弱小等问题。

\section{参考文献:}

[1] 王艳红. 基于 OpenCV 的运动目标检测与跟踪算法的研究 [D]. 杭州: 杭州电子科技大学, 2014.

WANG Yanhong. Research of Moving Object's Detection and Tracking Based on OpenCV[D]. Hangzhou: Dianzi University, 2014.

[2] 陆福星, 陈忻, 陈桂林, 等. 背景自适应的多特征融合的弱小目标检 测[J]. 红外与激光工程, 2019, 48(3): 277-283.
LU Fuxing, CHEN Xi, CHEN Guilin, et al. Dim and small target detection based on background adaptive multi-feature fusion[J]. Infrared and Laser Engineering, 2019, 48(3): 277-283.

[3] Kim S, YANG Y, Lee J, et al. Small target detection utilizing robust methods of the human visual system for IRST[J]. Journal of Infrared, Millimeter, and Terahertz Waves, 2009, 30(9): 994-1011.

[4] WANG X, LV G, XU L. Infrared dim target detection based on visual attention[J]. Infrared Phys. Techn., 2012, 55(6): 513-521.

[5] CHEN C P, LI H, WEI Y, et al. A local contrast method for small infrared target detection[J]. IEEE T. Geosci. Remote, 2013, 52(1): 574-581.

[6] XIE K, FU K, ZHOU T, et al. Small target detection based on accumulated center-surround difference measure[J]. Infrared Phys. Techn., 2014, 67: 229-236.

[7] ZHANG W, CONG M, WANG L. Algorithms for optical weak small targets detection and tracking[C]//International Conference on Neural Networks and Signal Processing IEEE, 2003: DOI: 10.1109/ ICNNSP.2003.1279357.

[8] 周苑, 张健民, 林晓. 基于加权 LoG 算子的红外弱小目标检测方法研 究[J]. 应用光学, 2017, 38(1): 114-119. 
ZHOU Yuan, ZHANG Jianmin, LIN Xiao. Infrared small target detection using weighting LoG operator[J]. Journal of Applied Optics, 2017, 38(1): 114-119.

[9] 韩金辉. 基于人类视觉特性的复杂背景红外小目标检测研究[D]. 武 汉：华中科技大学, 2016 .

HAN Jinhui. Infrared Small Target Detection under Complex Background Based on Human Visual System[D]. Wuhan: Huazhong University of Science and Technology, 2016.

[10] HAN J, LIANG K, ZHOU B, et al. Infrared small target detection utilizing the multiscale relative local contrast measure[J]. IEEE Geosci. Remote S., 2018, 15(4): 612-616.

[11] QIN Y, LI B. Effective infrared small target detection utilizing a novel local contrast method[J]. IEEE Geosci. Remote S., 2016, 13(12): 1890-1894.

[12] SHI Y, WEI Y, YAO H, et al. High-boost-based multiscale local contrast measure for infrared small target detection[J]. IEEE Geosci. Remote S., 2017, 15(1): 33-37.

[13] CAO Y, LIU R, YANG J. Small target detection using two-dimensional least mean square(TDLMS) filter based on neighborhood analysis[J]. International Journal of Infrared and Millimeter Waves, 2008, 29(2): 188-200.

[14] BAI X, ZHOU F. Analysis of new top-hat transformation and the application for infrared dim small target detection[J]. Pattern Recogn., 2010, 43(6): 2145-2156.

[15] GAO C, MENG D, YANG Y, et al. Infrared patch-image model for small target detection in a single image[J]. IEEE T. Image Process., 2013, 22(12): 4996-5009.

[16] LI Y, LI P, SHEN Q. Real-time infrared target tracking based on 11 minimization and compressive features[J]. APPL. OPTICS, 2014, 53(28): 6518-6526. 